\title{
Canine copper-associated hepatitis: A retrospective study of 17 clinical cases
}

\author{
Anthony Rodrigues ${ }^{1}$, Rodolfo O. Leal ${ }^{2 *}$, Maud Girod ${ }^{3}$, Claire Dally ${ }^{4}$, Emmanuel Guery ${ }^{4}$, Eymeric Gomes ${ }^{1}$ and \\ Juan Hernandez ${ }^{5}$ \\ ${ }^{1}$ Centre Hospitalier Vétérinaire Frégis, Arcueil, France \\ ${ }^{2}$ CIISA - Centro de Investigação em Sanidade Animal, Faculdade de Medicina Veterinária, \\ Universidade de Lisboa, Lisbon, Portugal \\ ${ }^{3}$ DFCvet, Rennes, France \\ ${ }^{4}$ LAPVSO - Laboratoire d'Anatomie Pathologique Vétérinaire du Sud-Ouest, Toulouse, France \\ ${ }^{5}$ Nantes-Atlantic College of Veterinary Medicine and Food Sciences, Nantes, France
}

\begin{abstract}
Background: Copper-associated hepatitis (CAH) is a well-documented chronic hepatic disease in dogs. In some breeds, the disease results from an inherited defect in copper metabolism. In others, it is unclear whether its acummulation is a primary or secondary condition. Reports of copper accumulation in dog breeds that are not genetically predisposed are increasing.

Aim: To describe the epidemiology, clinical and laboratory findings, liver biopsy techniques, and treatment response in dogs with CAH.

Methods: A retrospective study was performed, drawing upon medical records from CAH dogs at a Veterinary Referral Hospital in Paris, France. The diagnosis of CAH had been confirmed in these patients by positive rhodanine staining of hepatic tissue obtained through biopsy. Medical records were mined for the following data: age at presentation, sex, breed, chief presenting complaints, abdominal ultrasound (US) findings, and rhodanine staining pattern.

Results: A total of 17 dogs were included in the study. Median age at presentation was 8-year old (4-11). No sex predisposition was found. Terriers (4/17) and German Shepherd Dogs (GSD, 3/17) were overrepresented. American Staffordshire Terriers and Beauceron had not previously appeared in case reports on CAH; two of each breed were identified in this study. Clinical signs of affected dogs were non-specific. An incidental identification of increased liverenzymes was observed in 5/17 dogs. A heterogeneous, mottled liver was frequently described (5/17) on abdominal US. Liver biopsies were performed by US-guided percutaneous approach in 10/17 dogs, laparoscopy and laparotomy in $6 / 17$ and $1 / 17$, respectively. The rhodanine staining pattern was centrilobular (zone 3 ) in 8/17 dogs and periportal (zone 1) in $3 / 17$ dogs. The pattern was considered multifocal in $6 / 17$ dogs.

Conclusion: Increased liver enzymes may be the only clinical finding in dogs with copper-associated hepatitis, reflecting the silent progression of this disease. Centrilobular pattern of rhodanine staining was observed in the majority of cases suggesting the primary condition of the disease. Results of this study are consistent with the current literature, which reports that terriers and GSD are predisposed to CAH. This is the first description of CAH in Beauceron and American Staffordshire Terrier dogs.

Keywords: American Staffordshire Terrier, Beauceron, Copper-associated hepatitis, Liver biopsy, Rhodanine.
\end{abstract}

\section{Introduction}

Copper-associated hepatitis $(\mathrm{CAH})$ is a condition that is characterized by an abnormal accumulation of copper granules within hepatocytes, causing oxidative damage and inflammation (Fieten and Rothuizen, 2013). CAH is the most common cause of toxic chronic hepatitis in dogs (Webster et al., 2019). CAH can be divided into two broad categories, based upon its underlying cause. Primary $\mathrm{CAH}$ has a foundation in faulty genetics, and results from a defect in copper metabolism. As such, primary CAH is similar to Wilson's disease in humans (Roelen, 2013). The Bedlington Terrier, Doberman Pinscher, Dalmatian, Labrador Retriever, Cocker Spaniel, West Highland White Terrier, and Skye Terrier are predisposed to this type of CAH (Bexfield et al.,
2012; Webster et al., 2019). By contrast, secondary $\mathrm{CAH}$ results from an obstruction of bile flow that prevents the excretion of copper or excessive intake of this compound (Smedley et al., 2009).

Both primary and secondary CAH lead to increased levels of intra-hepatic copper although these levels appear to be higher in primary CAH (Hoffmann, 2009; Cedeño et al., 2016).

Although primary $\mathrm{CAH}$ predominates in the current veterinary medical literature, reports of non-genetically predisposed dogs (such as German Shepherds and Cocker Spaniels) are on the rise (Strickland et al., 2018). This raises concern that environmental exposure to some unknown factor is playing a role in the development of CAH (Johnston et al., 2013; 
Strickland et al., 2018). Nowadays, CAH seems to be a multifactorial disease.

Age and sex predisposition have been reported for specific breeds. In particular, female Labrador retrievers and Doberman pinschers appear to be predisposed (Hoffmann et al., 2006; Hoffmann, 2009). Young adult Labrador retrievers are the most impacted age group, with the onset of primary $\mathrm{CAH}$ occurring at a median age of 6-year old (Dirksen, 2016).

Clinical manifestations are non-specific and include anorexia or lethargy. Alternatively, clinical manifestations may also concentrate on the gastrointestinal tract, including vomiting, diarrhea, or melena. Patients may present with icterus or ascites. Clients may describe historical findings that are suggestive of polyuria and polydipsia. Neurological signs can also be present (Hoffmann et al., 2006; Hoffmann, 2009; Fieten and Rothuizen, 2013; Webster et al., 2019).

Liver biopsies are required for definitive diagnosis of CAH (WSAVA (Liver Standardization Group), 2006; Fieten and Rothuizen, 2013; Webster et al., 2019). Biopsies are also essential to assess the patient's prognosis and customize a treatment plan. Early diagnosis is preferred so as to slow, if not prevent, progression of the disease to the end stage of cirrhosis (Fieten and Rothuizen, 2013). This is achieved by addressing the underlying cause through therapeutic interventions that support liver function, and by preventing possible complications that may hinder quality of life. Therapeutic options include copperchelating drugs (e.g., penicillamine and trientine), copper-uptake restrictors (e.g., zinc), and cytoprotective compounds (e.g., S-Adenosylmethionine, silymarin, ursodeoxycholic acid, and vitamin E) (Fieten and Rothuizen, 2013).

Although $\mathrm{CAH}$ tends to occur in pure-breed dogs, several epidemiological factors are still unclear and it is believed that other less common breeds can also be affected (Fieten and Rothuizen, 2013; Webster et al., 2019). In fact, the current epidemiology of CAH is based on data from previous studies conducted in the Netherlands, United Kingdom, and United States of America, stressing the need to augment these findings with data from other countries (Bexfield et al., 2012; Roelen, 2013; Webster et al., 2019).

This retrospective study aims to describe the epidemiology, clinical and laboratory findings, diagnostic, and therapeutic interventions in dogs diagnosed with $\mathrm{CAH}$ at a Veterinary Referral Hospital in Paris, France.

\section{Material and Methods}

Medical records were obtained from a Veterinary Pathology Laboratory in France (LAPVSOLaboratoire D'Anatomie Pathologique Vétérinaire du Sud-Ouest, Toulouse, France). These were mined for all data corresponding to canine liver samples that tested positive to rhodanine staining, coming from a Veterinary Referral Hospital in Paris (Centre Hospitalier Vétérinaire Fregis, Arcueil, France), between May 2010 and March 2017. The corresponding medical records were obtained from the same referral center for a more complete database.

The information gathered from the medical records included age, sex, breed, presenting complaint(s), clinical sign(s), clinicopathologic data, ultrasound (US) findings, biopsy collection method, histopathological results, treatment, and follow-up.

Blood analysis consisted of: a complete blood count (CBC) and biochemistry profile that included alanine aminotransferase (ALT), alkaline phosphatase (ALP), gamma-glutamyl transpeptidase (GGT) and aspartate aminotransferase (AST), total proteins, albumin, glucose, creatinine blood urea nitrogen, and electrolytes (sodium, potassium, and chloride). If sequential blood analyses were performed on the same patient, then the results nearest to the date of first presentation, prior to initiation of treatment, were selected. In the face of increased hepatobiliary enzymes, patients were classified into one of three categories, relatively to the disparity with the reference values: mild, $<5$ times the upper limit; moderate, 5-10 times the upper limit, and marked, > 10 times the upper limit (Center, 2007).

Biopsy collection method was detailed and correlated with the respective quality of the sample. Data concerning quality of sample was based on the histopatology report and the subjective assessment performed by the pathologist being defined as poor, acceptable or good.

Hematoxyline-Eosine and Rhodanine staining were performed on all biopsy samples. The latter product stains intrahepatic copper granules, allowing for a qualitative assessment of hepatic copper accumulation (Johnston et al., 2013). CAH was classified as periportal (zone 1), centrilobular (zone 3) or multifocal according to rhodadine staining pattern within the hepatic acinus. In detail, hepatic acinus is divided into three zones according to the distance from oxygenated blood. Zone 1 corresponds to the periportal region, zone 3 refers to the acinus periphery, and zone 2 is interspersed inbetween them (Mescher, 2016). CAH was then classified into periportal or centrilobular if rhodamine mainly stained zone 1 or zone 3, respectively. When no clear prevalence was identified and rhodanine was scattered within the hepatic architechture, the pattern was considered as multifocal.

Descriptive statistical analysis was performed using commercial software IBM, SPSS Statistics and Microsoft Excel 2016. A chi-squared test was performed to assess for gender predisposition. A $p$ value $<0.05$ was considered as significant.

Ethical approval

Due to the retrospective nature of the study, no ethical approval was obtained. 


\section{Results}

A total of 17 dogs were included in this study. The median age at presentation was 8 years (range, 4-11 years). Eleven dogs were female $(65 \%-6$ of which spayed and 5 intact) and 6 were male $(35 \%-2$ castrated and 4 intact). No relevant statistical difference was found $(p=0.23)$. Multiple breeds were identified (Fig. 1): 5/17 Shepherds (3 GSD and 2 Beauceron), 4/17 Terriers (2 American Staffordshire Terrier, 1 Jack Russel Terrier and 1 West Highland White Terrier), 4/17 spaniels (2 Cavalier King Charles Spaniel and 2 Cocker spaniel), 2/17 Labrador retriever, 1/17 Dalmatian and
1/17 Mongrel. One dog had been previously diagnosed with hypoadrenocorticism and hypothyroidism and was treated with fludrocortisone and levothyroxine; another dog had been diagnosed with a mitral valve regurgitation (ACVIM stage $C$ ) and was treated with pimobendan, spironolactone, torasemide, and benazepril.

The chief complaints are summarized in Figure 2 and included: gastrointestinal signs (13/17), blood abnormalities (6/17), neurological signs (1/17), jaundice $(2 / 17)$, and ascites (1/17).

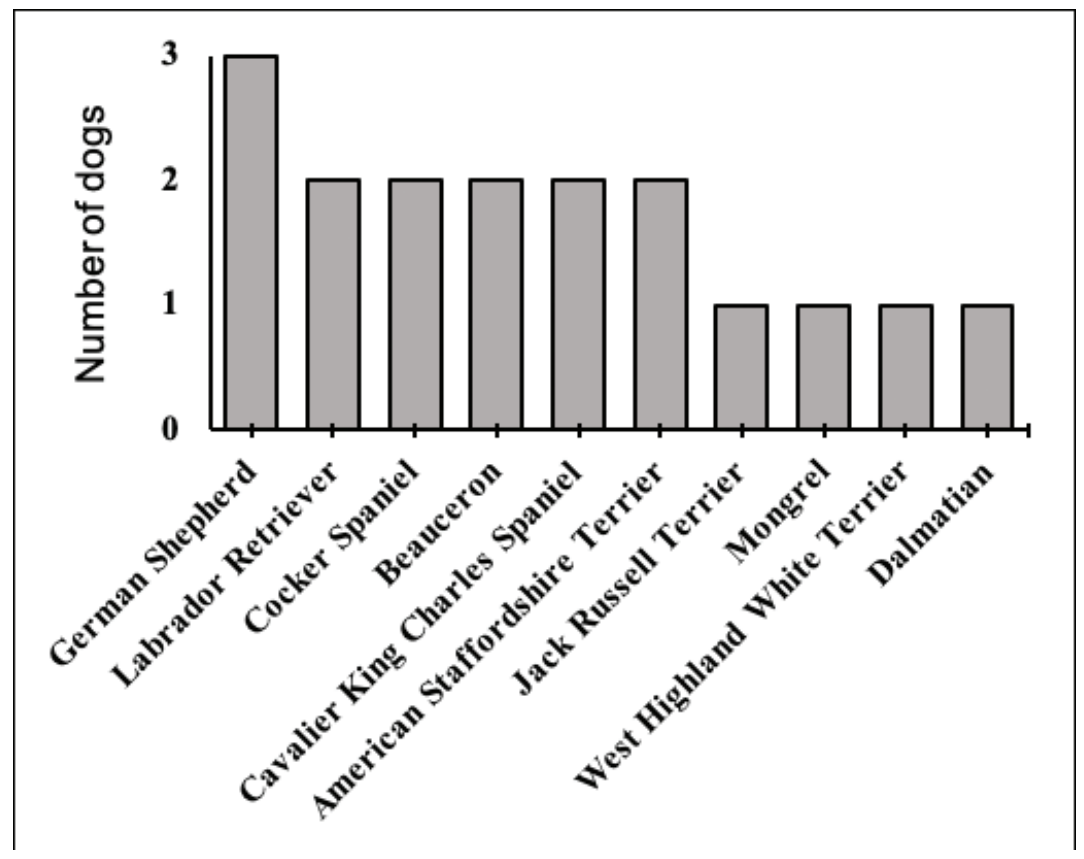

Fig. 1. Distribution of dog breeds diagnosed with copper-associated hepatitis between May 2010 and March 2017 in a French Veterinary Referral Center.

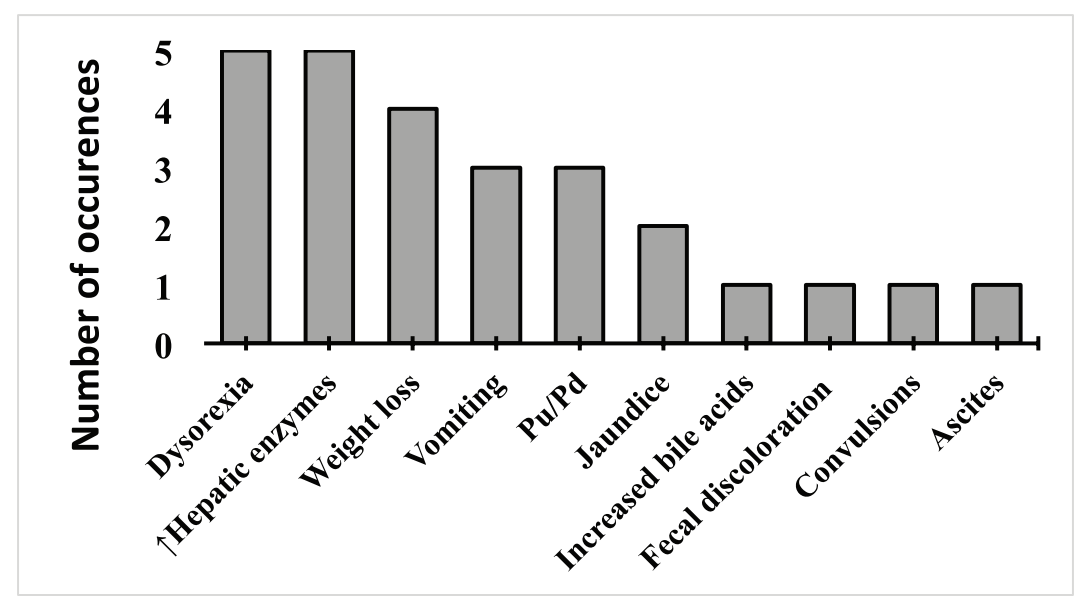

Fig. 2. Main complaints of dogs diagnosed with copper-associated hepatitis between May 2010 and March 2017 in a Veterinary Referral Hospital from Paris region (France). 
$\mathrm{CBC}$ results from 7 dogs were available and all parameters were within the reference range. Although incomplete in several cases, biochemistry profiles were available in 16/17 dogs. Fifteen out of 15 dogs presented with increased ALT (mild, $n=2$; moderate, $n$ $=4 ;$ marked =9), 12/12 with increased ALP (mild, $n=$ 5; moderate, $n=3$; marked, $n=4)$, 5/6 with increased GGT (mild, $n=2$; moderate, $n=3$ ) and $1 / 1$ with increased AST (mild, $n=1$ ). Total protein was decreased in 7/12 dogs, 5 of which were also hypoalbuminemic. The remaining parameters were unremarkable.

Abdominal US was performed in all dogs in this study. Findings included an heterogenous mottled liver (5/17), hepatomegaly (4/17), hypoechoic liver nodules (3/17), microhepatica (3/17), and hepatic lymphadenomegaly (3/17). Other findings included: a distended gallbladder $(1 / 17)$, intra and extra-hepatic choledocholithiasis $(1 / 17)$, increased pancreatic size (1/17), thickened gastric wall $(2 / 17)$, splenomegaly (1/17), intestinal parietal segmented lesions that were suggestive of chronic inflammation (1/17), mild peritoneal effusion $(1 / 17)$ and mineralization of the adrenal glands (1/17). Four dogs had no observable ultrasonographic alteration of the liver. An angioscan was performed on one dog with elevated biliary acids and a history of seizures but no vascular abnormalities were found.

Ten out of the 17 biopsies were performed using a percutaneous approach (14 gauge true-cut needle biopsy), laparoscopy (using cup biopsy forceps) was performed in 6/17 and laparotomy was done in $1 / 17$. The number of samples that were obtained ranged from one to two samples for cases in which true-cut needle biopsy was performed; and from one to five in all other techniques. The quality of samples obtained by percutaneous approach was classified as poor in $5 / 10$, acceptable in $2 / 10$, good in $2 / 10$, and very good in $1 / 10$. Those obtained by laparoscopy were mostly acceptable ( poor $=2$; acceptable $=3$; good $=1$ ), and the surgical sample was estimated very good. The rhodanine staining pattern was centrilobular (zone 3) in 8/17 dogs (Figs. 3 and 4) and periportal (zone 1) in 3/17 dogs. In $6 / 17$ dogs, the pattern was considered as multifocal.

The information about the prescribed treatment was available for 11 out of the 17 clinical cases. The most frequent treatment was S-Adenosylmethionine (8/11) followed by a prescription diet (7/11), penicillamine (6/11), and corticosteroids (4/11). Additionally, two dogs received ursodeoxycholic acid and vitamin E, and one dog received azathioprine and maropitant. Of those, only $8 / 11$ returned to the referral center for follow-up. Overall, 4/8 dogs showed a clinical improvement. In the first 2 months of treatment, there was no decrease in liver enzymes in $3 / 8$ dogs and clinical improvement was only observed in one of them. Between 2 and 21 months after the onset of medical treatment, four out of these eight patients demonstrated a decrease in blood liver enzymes. Only one of these

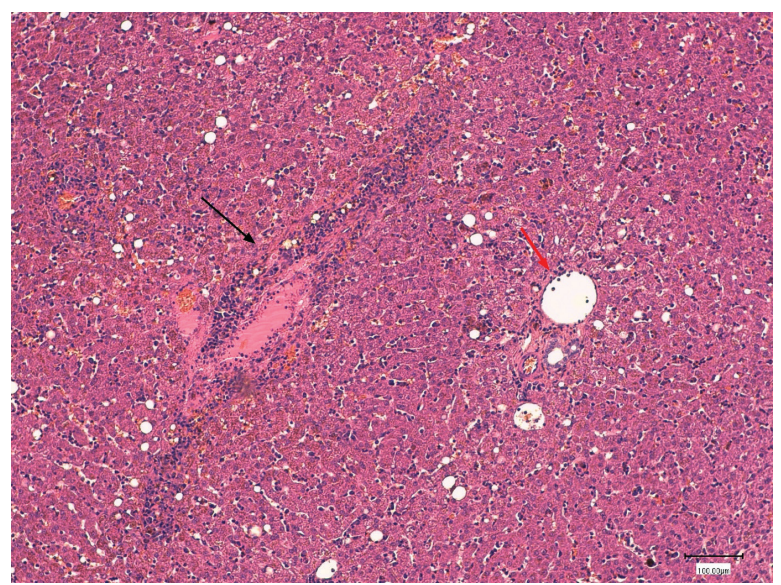

Fig. 3. Histological section of canine liver. Haematoxylineosin stain. Magnification $\times 100$, bar $=100,100 \mu \mathrm{m}$. Hepatitis with centrolobular zone infiltrated by lymphocytes, plasma cells and a few neutrophils (black arrow). Normal portal space (red arrow).

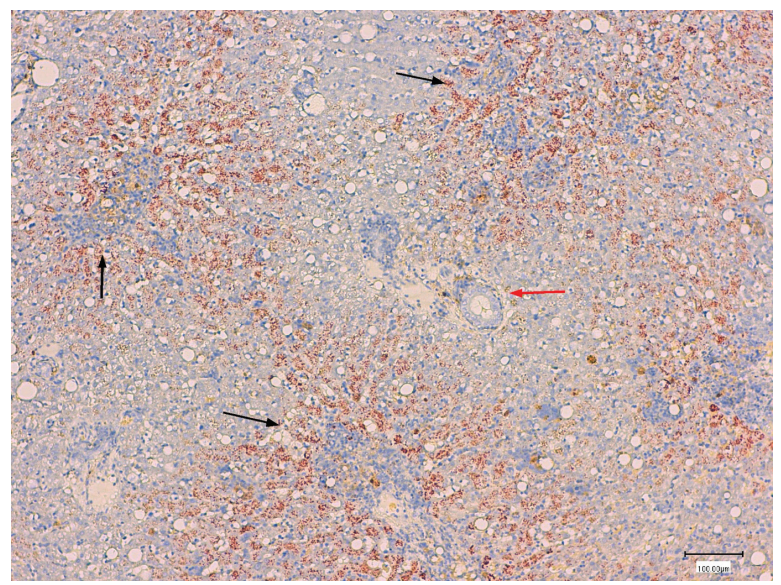

Fig. 4. Histological section of canine liver. Rhodanine stain. Magnification $\times 100$, bar $=100 \mu \mathrm{m}$. Copper accumulation around the centrolubular spaces (black arrows) with relative sparing of the periportal area (red arrow).

dogs submitted to a follow-up liver biopsy, 4 months later, which revealed persistent histological signs of inflammation, albeit less marked. One case underwent a cholecystoduodenostomy due to choledocholithiasis. This procedure reportedly led to clinical improvement and a concurrent decrease in liver-enzymes, which became borderline-normal.

\section{Discussion}

The present study assessed the main epidemiological factors, clinical and laboratory findings, liver biopsy technique, and response to treatment in 17 dogs diagnosed with $\mathrm{CAH}$. Concerning breed predisposition, this study is in agreement with previous ones reporting 
an over representation of Terriers and German Shepherd Dogs. To the author's knowledge, this is the first study describing CAH in four cases of two breeds not yet reported: American Staffordshire Terriers and Beaucerons. As the majority of the reported cases are from the United Kingdom, Netherlands, or United States, this study shows a different trend in affected breeds (Bexfield et al., 2012; Roelen, 2013; Webster et al., 2019). American Staffordshire Terrier is somewhat expected as the disease is well described in Terrier's group. Beauceron is a french breed of the pastoral group, relatively common in France. Originating from the region of Beauce (France), it is one of the native breeds of the country, with the highest population size (Leroy et al., 2006). Similarly to German Shepherd Dogs, Beauceron takes part of the Sheepdogs and Cattledogs, according to the World Canine Organization (Féderation Cynologique Internationale). Although to author's knowledge, there is no truly relationship inbetween these breeds, they have common phenotypic and behavioral findings, suggesting a possible common genetic basis for CAH predisposition. Despite the low number of identified cases, this study raises the concern of a potential new-breed predisposition emphasizing the need of a medical exploration in case of increased liverenzymes. Overall, this study not only reinforces the overexpression of $\mathrm{CAH}$ in Terriers but also increases the spectrum of documented breeds affected with CAH. Although gender predisposition was not statistically relevant, the fact that $\mathrm{CAH}$ were more frequently identified in females and the median age of 8 years are in agreement with previous studies (Hoffmann, 2009; Dirksen, 2016).

Clinical signs were non-specific, with five cases only demonstrating increased liver enzymes, reflecting the silent progression of $\mathrm{CAH}$ and capacity of the liver to withstand large amounts of oxidative stress.

Regarding clinicopathologic data, all patients demonstrated an increase in one or more liver enzymes. In particular, patients tended to experience a marked increase in ALT, thus substantiating the presence of parenchymal disease. Two-thirds of affected dogs demonstrated hepatic changes on abdominal US. However, these changes were not specific. In agreement with previous studies, these results support that biochemical analysis are the most pertinent first line complementary exam to assess hepatic parenchymal disease (Webster et al., 2019). In contrast, ultrasonography can be useful to assess the nature of those lesions as well as the need for more invasive diagnostic procedures, such as liver biopsies. In these animals, different ultrasonography findings were observed and only in four dogs this exam was unremarkable. Nonetheless, ultrasonography is a valuable complementary exam in the majority of dogs, reinforcing the need of liver sampling.
In fact, histopathology of liver tissue remains the most concise method to confirm the accumulation of copper and presence of hepatitis (WSAVA (Liver Standardization Group), 2006; Webster et al., 2019). The percutaneous needle biopsy was the most used technique in this study, followed by cup wedge biopsy through laparoscopy. Previous studies have shown that the first one should not be the main choice due to the suboptimal sized samples that it provides and consequent friability, resulting in low-quality biopsies (Cole et al., 2002). The information gathered from the histopathological reports conducted in this study matched these observations since $50 \%$ of biopsies performed with a percutaneous needle were classified as poor, as opposed to $20 \%$ for laparoscopic wedge biopsy and none for the surgical approach. However, it has recently been demonstrated that needle samples, cup samples and punch liver samples tend to provide similar histopathologic diagnoses since they both deliver a sufficient number of portal triads, and that more important than the technique used is the number of samples collected (Kemp et al., 2015).

In general, techniques providing larger biopsies should be favoured and when not feasible, the collection of multiple samples should be made. Based on this study, results, and on the recent literature, it is then reasonable to assume that when a biopsy is taken, the clinician should consider the technique providing larger biopsies and, when it is not feasible, multiple samples should be done. Overall, it seems more pratical to consider surgical or laparoscopy approach in order to increase the chances of acquiring good samples and a reasonable ammount of tissue for a more reliable histological diagnosis.

Copper accumulation was confirmed in all clinical cases through rhodanine staining. The centrilobular stain pattern observed in the majority of dogs emphasizes the primary condition of the disease. This pattern is a recurrent finding in breeds in which the hereditary cause has been confirmed (Hoffmann et al., 2006). Periportal copper accumulation was observed in three dogs. This could be explained by the presence of periportal inflammation, hindering bile flow in this region. All these signs supports cholestasis, meaning that the accumulation of copper in those dogs, was likely to be secondary to the obstruction of bile flow. Finally, the multifocal copper accumulation was usually attributed by the pathologists to the overall low quality of the samples provided (small size, friability) and to a lesser degree to the marked structural modifications due to fibrosis. It can be concluded that copper can be distributed preferentially in zone 1 or 3 , depending on the subjacent cause and occasionally be judged as multifocal due to either poor biopsy collection or advanced stage of the disease. 
A main limitation of this work is the absence of routine copper quantification in all the patients. This can be explained by the need to collect a large piece of liver tissue, which translates into a more invasive procedure, associated with inherent risk and elevated costs. Even if copper quantification could eventually have been performed in samples collected by laparotomy or laparoscopy, the cost and sub-optimal shipment conditions limited its feasibility. Although it was suggested, owners declined to pursue this option. This complicates followup decisions about treatment options, for instance, whether or not to initiate aggressive treatments, such as chelation therapy, or to advise a change of diet (Fieten and Rothuizen, 2013). Furthermore, the duration of treatment depends upon the copper concentration and its excessive duration could rapidly become deleterious leading to eventual copper deficiency on long-term follow-up. Efforts should then be made to advise the owners of the importance of this additional test and potential risks that are associated with not performing it.

Another limitation of this study is the lack of data concerning treatment and follow-up. As most of these cases were referrals, several were lost to follow-up after the definitive diagnosis. Further prospective studies are needed to evaluate if clinical response is similar in dogs with CAH.

In summary, this study confirmed that the silent progression of $\mathrm{CAH}$ is a real phenomenon and that early detection presents a clinical challenge. The presence of subtle clinical signs or isolated increased liver-enzymes should not be ignored, as they can be the early reflection of an ongoing inflammatory process. This study also permitted to broaden the spectrum of reported breeds, as to the authors knowledge, this is the first report describing $\mathrm{CAH}$ in Beauceron and American Staffordshire Terrier dogs.

\section{Acknowledgments}

The authors would like to thank the Internal Medicine Team from CHV Fregis and the LAPVSO team for the enrolement in these cases and to Dr. Ryane Englar for reviewing the manuscript.

\section{Conflict of interest}

The Authors declare that there is no conflict of interest Authors contribution

A.Rodrigues were involved on the data collection and preparation of the manuscript; R.O. Leal, M. Girod, and J. Hernandez supervised the preparation of the manuscript, contributing for the scientific background and discussing its main time points; E.Guery and C.Dally were involved on data collection at LAPVSO contributing for the histopathology results, figures and pathology feedback; E.Gomes reviewed all the imaging database, contributing for the US results feedback.

\section{References}

Bexfield, N.H., Buxton, R.J., Vicek, T.J., Day, M.J., Bailey, S.M., Haugland, S.P., Morrison, L.R., Else,
R.W., Constantino-Casas, F. and Watson, P.J. 2012. Breed, age and gender distribution of dogs with chronic hepatitis in the United Kingdom. Vet. J. 193, 124-8.

Cedeño, Y., López-Alonso M. and Miranda M. 2016. Hepatic concentrations of copper and other metals in dogs with and without chronic hepatitis. J. Small Anim. Pract. 57, 703-9.

Center, S.A., 2007. Interpretation of liver enzymes. Vet. Clin. Small Anim. 37, 297-333.

Cole, T.L., Center, S.A., Flood, S.N., Rowland, P.H., Valentine, B.A., Warner, K.L. and Erb, H.N. 2002. Diagnostic comparison of needle and wedge biopsy specimens of the liver in dogs and cats. J. Am. Vet. Med. Assoc. 220, 1483-90.

Dirksen, K. 2016. Copper-associated hepatitis in dogs; pathogenesis, diagnosis and treatment. Ph.D. Thesis, Department of Clinical Sciences of Companion Animals, Faculty of Veterinary Medicine, Utrecht University, Utrecht, Netherlands.

Fieten, H. and Rothuizen, J. 2013. Copper Associated Hepatitis (webchapter). In Kirk's current veterinary therapy XV. Eds., Bonagura, J.D. and Twedt, D.C. St. Louis, MO: Saunders.

Hoffmann, G. 2009. Copper-associated liver diseases. Vet. Clin. North Am. Small Anim. Pract. 39, 489511.

Hoffmann, G., van den Ingh, T.S.G. A. M., Bode, P. and Rothuizen, J. 2006. Copper-associated chronic hepatitis in Labrador Retrievers. J. Vet. Intern. Med. 20, 856-61.

Johnston, A.N., Center, S.A., McDonough, S.P., Wakshlag, J.J. and Warner, K.L. 2013. Hepatic copper concentration in Labrador Retrievers with and without chronic hepatitis: 72 cases (19802010). J. Am. Med. Assoc. 242, 372-80.

Kemp, S.D., Zimmerman, K.L., Panciera, D.L., Monroe, W.E., Leib, M.S. and Lanz, O.I. 2015. A comparison of liver sampling techniques in dogs. J. Vet. Intern. Med. 29, 51-57.

Leroy, G., Rognon, X., Varlet, A., Joffrin, C. and Verrier, E. 2006. Genetic variability in French dog breeds assessed by pedigree data. J. Anim. Breed. Genetics 123, 1-9.

Mescher, A.L. 2016. Junqueira's basic histology, 14th ed. New York, NY: McGraw-Hill Education.

Roelen, Y. 2013. Hepatic copper accumulation in Labrador Retriever and Wilson's disease - Honours Program 2012-2013. Department of Companion Animals - Department of Clinical Sciences of Companion Animals, Faculty of Veterinary Medicine, Utrecht University, the Netherlands (Thesis available online).

Smedley, R., Mullaney, T. and Rumbeiha, W. 2009. Copper-associated hepatitis in Labrador Retrievers. Vet. Pathol. 46, 484-90.

Strickland, J.M., Buchweitz, J.P., Smedley, R.C., Olstad, K.J., Schultz, R.S., Oliver, N.B. and Langlois, D.K. 
2018. Hepatic copper concentrations in 546 dogs (1982-2015). J. Vet. Intern. Med. 32, 1943-50.

Webster, C.R.L., Center, S.A., Cullen, J.M., Penninck, D.G., Richter, K.P., Twedt, D.C. and Watson, P.J. 2019. ACVIM consensus statement on the diagnosis and treatment of chronic hepatitis in dogs. J. Vet. Intern. Med. 33, 1173-200.
WSAVA (Liver Standardization Group). 2006. WSAVA: standards for clinical and histological diagnosis of canine and feline liver disease. Philadelphia, PA: Saunders. 